\title{
Mobile government: uma análise dos aplicativos estaduais como mediadores do relacionamento entre os cidadãos e os governos estaduais
}

\author{
Rodrigo Diniz Lara \\ Secretaria de Estado de Planejamento e Gestão de Minas Gerais \\ Marlusa Gosling \\ Universidade Federal de Minas Gerais (UFMG) \\ Izabela França Rodrigues \\ Secretaria de Estado de Planejamento e Gestão de Minas Gerais
}

Segundo a Pesquisa Nacional por Amostra de Domicílios (Pnad) 2015, 92,1\% dos domicílios brasileiros acessaram a internet por meio do telefone celular. Esses dados trouxeram oportunidades e desafios para a administração pública no sentido de utilizar a potencialidade do mobile government ( $\mathrm{m}$-gov) para a prestação de serviços. O objetivo geral é avaliar os aplicativos móveis dos governos estaduais e do Distrito Federal que centralizam a prestação de serviços, para verificar a aderência às melhores práticas de acordo com a literatura nacional e internacional para aprimorar o relacionamento com o cidadão. Apenas 10 unidades federativas do escopo pesquisado possuem um aplicativo que centraliza os serviços dos governos. O número de downloads também representa uma pequena parcela da população. Poucos aplicativos utilizam-se dos recursos tecnológicos típicos dos dispositivos móveis como GPS, câmera e avisos por push para aprimorar o relacionamento com o cidadão.

Palavras-chave: governo eletrônico, prestação de serviços, Estado e sociedade, administração estadual - Brasil

[Artigo recebido em 14 de fevereiro de 2018. Aprovado em 12 de junho de 2018] 
Mobile government: un análisis de las aplicaciones estatales como mediadores de la relación entre los ciudadanos y los gobiernos

Según la Encuesta Nacional por Muestra de Domicilios (Pnad) 2015, el 92,1\% de los domicilios brasileños accedieron a internet a través del teléfono celular. Estos datos trajeron oportunidades y desafíos para la administración pública para utilizar la potencialidad del mobile government (m-gov) para la prestación de servicios. El objetivo general es evaluar las aplicaciones móviles de los gobiernos estatales y del Distrito Federal que centralizan la prestación de servicios para verificar la adherencia a las mejores prácticas de acuerdo con la literatura nacional e internacional para mejorar la relación con el ciudadano. Sólo 10 unidades federativas del ámbito investigado poseen una aplicación que centraliza los servicios de los gobiernos. El número de descargas también representa una pequeña parte de la población. Pocas aplicaciones se utilizan de los recursos tecnológicos típicos de los dispositivos móviles como GPS, cámara y avisos por push para mejorar la relación con el ciudadano.

Palabras clave: gobierno electrónico, prestación de servicios, Estado y sociedad, administración de los estados - Brasil

Mobile government: an analysis of state applications as mediators ofthe relationship between citizens and governments

According to the National Household Sample Survey (Pnad) 2015, 92.1\% of Brazilian households accessed the internet through cell phones. These data have brought opportunities and challenges for the public administration to use the potential of mobile government (m-gov) to deliver services. The overall objective is to evaluate the mobile applications of the state and Federal District governments that centralize the provision of services to verify adherence to best practices according to the national and international literature to improve the relationship with the citizen. Only 10 federative units of the researched scope have an application that centralizes government services. The number of downloads also represents a small portion of the population. Few applications use the typical technology features of mobile devices such as GPS, camera and push notifications to enhance the relationship with the citizen.

Keywords: electronic government, service delivery, State and society, state administration - Brazil 


\section{Introdução}

O advento das tecnologias da informação e comunicação (TIC) e o aumento da sua utilização em nível mundial têm propiciado o alargamento dos debates em torno das interações promovidas por essas tecnologias. Nas últimas décadas, as mudanças geradas pela expansão das TIC resultaram na reconfiguração de todo o ambiente em que estão inseridos os atores sociais, com novos desafios e oportunidades (ARAúJo, 2005).

Castells (2003, p. 68) ressalta a importância do advento de tal inovação:

Diferentemente de qualquer outra revolução, o cerne da transformação que estamos vivendo na revolução atual refere-se às tecnologias da informação, processamento e comunicação. A tecnologia da informação é para esta revolução o que as novas fontes de energia foram para as revoluções industriais sucessivas, do motor a vapor à eletricidade, aos combustíveis fósseis e até mesmo à energia nuclear.

Segundo Medeiros e Guimarães (2004), a "sociedade em rede", fruto desse desenvolvimento tecnológico, produz constantemente novas demandas ao setor público, buscando aprimorar as interações com o Estado. Diante de tais mudanças, os governos precisaram adaptar-se a esse novo ambiente complexo, para operar em novas realidades globalizadas, construindo uma nova relação entre governo e cidadãos, baseada nas tecnologias da informação e comunicação (TIC). Ferguson (2002) afirma que a disseminação da tecnologia na vida do cidadão moderno impulsiona a utilização pelos governos em todos os aspectos relacionados ao atendimento e à administração pública.

Nesse mesmo sentido, Medeiros e Guimarães (2004) destacam que a introdução dessas novas tecnologias na sociedade altera a abordagem da gestão pública, uma vez que os cidadãos e empresas querem cada vez mais ter acesso ao governo de forma rápida e facilitada. Essa utilização das TICs pela gestão pública tem sido chamada de governo eletrônico ou e-Gov, que pode ser entendido como a prestação de informações e a disponibilização de serviços governamentais por meio da internet (MEDEIROS; GUIMARÃES, 2006).

Uma nova perspectiva do governo eletrônico que ganha cada vez mais espaço no ambiente governamental é governo eletrônico móvel ou m-gov (Mobile Government), com a utilização das tecnologias de informação e comunicação (TIC) para a prestação de serviços públicos por meio de plataformas móveis como celulares, smartphones e tablets. O número de assinaturas de telefones móveis no mundo passou de 2,2 bilhões em 2005 para 7,1 bilhões em 2015, sendo que 3,2 
bilhões desses utilizam internet. Esse aumento representa mais de 43 por cento da população mundial total, dos quais 2 bilhões são de países em desenvolvimento (ORGANIZAÇÃo dAS NAÇÕES UNIDAS, 2016).

O uso de dispositivos móveis pela população brasileira tem se intensificado nos últimos anos, o que tem ocasionado várias mudanças na sociedade. Segundo dados da Pesquisa Nacional por Amostra de Domicílios (Pnad) 2015 (BRASIL, 2016), $92,1 \%$ dos domicílios brasileiros acessaram a internet por meio do telefone celular, enquanto $70,1 \%$ dos domicílios o fizeram por meio do microcomputador. Os dados da Pnad 2015 também demonstram que o percentual de pessoas que acessaram a internet alcançou $57,5 \%$ da população de 10 anos ou mais de idade, o que corresponde a 102,1 milhões de pessoas.

Com o avanço da telefonia móvel, que se tornou o principal meio de acesso à internet nos domicílios dos brasileiros, o m-gov trouxe uma nova perspectiva ao governo eletrônico. Essas mudanças trouxeram oportunidades e desafios para a administração pública no sentido de utilizar a potencialidade dessa nova tecnologia para a prestação de serviços públicos.

Segundo Pimentel (2009, p. 15) o m-gov é uma modalidade de e-gov que tem como "objetivo criar uma ligação entre os sistemas do governo e os cidadãos, através dos seus aparelhos celulares. Suas vantagens em relação às aplicações tradicionais do e-gov, que normalmente são aplicações web, são a mobilidade e o uso de redes sem fio para trocas de dados".

Kushchu e Kuscu (2003, p.3, tradução nossa) definem o m-gov como uma:

(...) implementação envolvendo a utilização de todos os tipos de tecnologia móvel e sem fio, serviços, aplicativos e dispositivos para melhorar os benefícios para as partes envolvidas no governo eletrônico, incluindo cidadãos, empresas e todas as unidades do governo.

É importante frisar que o m-gov não pode ser entendido como um substituto do e-gov. Na verdade, o m-gov é um complemento do governo eletrônico (BERBE, 2014). Para os autores Allazo, Sablón e lano (2009), o m-gov é um subconjunto do e-gov no qual a utilização da informação e tecnologias possibilitam inovar as atividades das organizações focadas no setor público. As Organizações das Nações Unidas (2016) destacam que os serviços on-line estão cada vez mais inovadores por meio do m-gov, atendendo as necessidades individuais dos cidadãos. A lógica do m-gov é prestar o serviço "a qualquer hora e em qualquer lugar" (ALLAZO; SABLóN; IANO, 2009; ORGANIZAÇÃO DAS NAÇÕES UNIDAS, 2016)

Berbe (2014) destaca que o m-gov emerge como uma tendência e uma nova fronteira para a gestão pública, incrementando o potencial de transformação na 
prestação de serviços públicos. Três fatores que podem motivar a ampliação da oferta de serviços de m-Gov pelos governos, como por exemplo: maior disponibilidade, escalabilidade e menores custos.

Apesar da propagação da internet móvel e dos benefícios do m-gov, um interessante estudo denominado eGovernment Benchmark 2017 - Taking stock of user-centric design and delivery of digital public services in Europe, produzido pela União Europeia (2017), destaca que as administrações públicas europeias não avançam na mesma proporção de implantação do m-gov. Em 2016, apenas 1 de cada 2 serviços estavam disponíveis on-line por meio de smarthphones ou tablets.

O objetivo geral deste trabalho é avaliar os aplicativos móveis dos governos estaduais e do Distrito Federal para verificar se eles estão aderentes às melhores práticas de mobile government ( $\mathrm{m}$-gov) para aprimorar o relacionamento com o cidadão. Os objetivos específicos são:

a. explorar a literatura nacional e internacional sobre m-gov e identificar critérios para avaliação de aplicativos;

b. realizar levantamento junto aos estados e ao Distrito Federal para identificar quais utilizam aplicativos móveis que agregam serviços públicos para se relacionar com o cidadão e que recursos utilizam;

c. identificar critérios que podem ser incorporados às metodologias levantadas de $\mathrm{m}$-gov a partir da avaliação de aplicativos nacionais e internacionais; e

d. realizar uma análise crítica destes aplicativos de governo em relação aos critérios levantados;

\section{Metodologia}

Para atingir os objetivos do trabalho, foi realizado inicialmente um levantamento junto à literatura nacional e internacional sobre critérios de avaliação de aplicativos m-gov.

A segunda etapa foi uma pesquisa e download de todos os aplicativos de m-gov dos Governos Estaduais e do Distrito Federal que utilizam a tecnologia Android disponíveis na loja Play Store. Optou-se por focar o estudo na tecnologia Android pois ela tem 93,2\% do mercado brasileiro de dispositivos móveis (CARVALHo, 2017). O número é referente à análise conduzida entre dezembro de 2016, janeiro e fevereiro de 2017 no país. Outra opção foi pesquisar aplicativos dos governos que reunissem mais de um serviço do governo. Dessa forma, não foi realizada a análise de aplicativos de órgãos específicos, como Detran. A pesquisa foi realizada em junho de 2017. 
Foram identificados dez aplicativos listados a seguir:

1. Bahia (SAC BA Mobile);

2. Espírito Santo (ES na Palma da Mão);

3. Goiás (VaptVupt);

4. Mato Grosso (MT Cidadão);

5. Minas Gerais (MG app);

6. Pará (Governo Digital Pará);

7. Paraíba (Governo da Paraíba);

8. Piauí (Gestão Transparente);

9. Rio Grande do Sul (RS Móvel); e

10. São Paulo (SP Serviços)

Aos critérios levantados na literatura foram acrescentadas as informações levantadas na loja Play Store com número de downloads, tamanho do aplicativo, nota do aplicativo na loja e a data de atualização. Outros critérios foram acrescentados com base na avaliação das funcionalidades e características levantadas nos aplicativos que não foram identificados na literatura. A junção dessas informações gerou o Quadro 1. Os critérios identificados foram agrupados nas seguintes categorias: ajuda e documentação, conteúdo, funcionalidade, informações sobre o aplicativo, relacionamento com o cidadão, segurança da informação, serviços e usabilidade.

Quadro 1 - Critérios Avaliados

\begin{tabular}{|llll|}
\hline Categoria & Critério & Descrição & Referência \\
\hline $\begin{array}{l}\text { Ajuda e } \\
\text { documentação }\end{array}$ & Ajuda & $\begin{array}{l}\text { Disponibilizar uma } \\
\text { opção de ajuda para } \\
\text { o usuário identificar } \\
\text { as respostas para as } \\
\text { principais dúvidas sobre } \\
\text { o aplicativo. }\end{array}$ & $\begin{array}{l}\text { Olibário, 2013; } \\
\text { Berbe, 2014 }\end{array}$ \\
\hline $\begin{array}{l}\text { Ajuda e } \\
\text { documentação }\end{array}$ & Termos de uso & $\begin{array}{l}\text { Apresentar as políticas } \\
\text { de uso do aplicativo. }\end{array}$ & Berbe, 2014 \\
\hline
\end{tabular}




\begin{tabular}{|c|c|c|c|}
\hline Conteúdo & Áreas de atuação & $\begin{array}{l}\text { Identificar as áreas dos } \\
\text { serviços oferecidos no } \\
\text { aplicativo: identificação } \\
\text { civil; achados e perdidos; } \\
\text { trabalho e emprego; } \\
\text { segurança; água e } \\
\text { esgoto; saúde; energia; } \\
\text { veículos e condutores; } \\
\text { ouvidoria; unidades; } \\
\text { servidor; pesquisa de } \\
\text { processos; educação; } \\
\text { legislação; empresa; } \\
\text { diário oficial; tributos; } \\
\text { agricultura; animais; } \\
\text { linha de ônibus; } \\
\text { transparência; meio } \\
\text { ambiente; notícias; } \\
\text { agenda }\end{array}$ & $\begin{array}{l}\text { Centeno, } \\
\text { Andrade e } \\
\text { Souza, } 2015\end{array}$ \\
\hline Conteúdo & $\begin{array}{l}\text { Conteúdo } \\
\text { classificados por } \\
\text { assunto }\end{array}$ & $\begin{array}{l}\text { Classificar o conteúdo } \\
\text { disponibilizado no } \\
\text { aplicativo conforme } \\
\text { as necessidades } \\
\text { dos usuários, e não } \\
\text { conforme a estrutura } \\
\text { governamental. }\end{array}$ & Berbe, 2014 \\
\hline Funcionalidade & Campo de busca & $\begin{array}{l}\text { Disponibilizar um campo } \\
\text { de busca no aplicativo } \\
\text { para o usuário buscar as } \\
\text { informações e serviços } \\
\text { oferecidos }\end{array}$ & $\begin{array}{l}\text { Neto, 2016; } \\
\text { Berbe, } 2014\end{array}$ \\
\hline Funcionalidade & $\begin{array}{l}\text { Utilização de } \\
\text { câmeras para } \\
\text { prestação do } \\
\text { serviço }\end{array}$ & $\begin{array}{l}\text { Permitir que o usuário } \\
\text { utilize a câmera do } \\
\text { celular para realização } \\
\text { de algum serviço. } \\
\text { Exemplo 1: o usuário } \\
\text { pode tirar uma foto e } \\
\text { anexá-la ao cadastro. } \\
\text { Exemplo 2: o cidadão } \\
\text { pode enviar uma } \\
\text { reclamação com foto } \\
\text { para ilustrar algum } \\
\text { tipo de problema } \\
\text { encontrado. }\end{array}$ & $\begin{array}{l}\text { Centeno, } \\
\text { Andrade e } \\
\text { Souza, 2015; } \\
\text { Berbe, 2014 }\end{array}$ \\
\hline
\end{tabular}




\begin{tabular}{|c|c|c|c|}
\hline Funcionalidade & $\begin{array}{l}\text { Utilização de GPS } \\
\text { para prestação do } \\
\text { serviço }\end{array}$ & $\begin{array}{l}\text { Utilizar geolocalização } \\
\text { (GPS) para aprimorar } \\
\text { a experiência de uso. } \\
\text { Exemplo 1: utilizar a } \\
\text { posição mostrada pelo } \\
\text { GPS do dispositivo para } \\
\text { indicar a aproximação } \\
\text { de ônibus numa } \\
\text { determinada parada. } \\
\text { Exemplo 2: permitir } \\
\text { que o usuário descubra } \\
\text { a distância para } \\
\text { chegar a uma praça de } \\
\text { atendimento do órgão } \\
\text { público. }\end{array}$ & $\begin{array}{l}\text { Centeno, } \\
\text { Andrade e } \\
\text { Souza, 2015; } \\
\text { Berbe, 2014 }\end{array}$ \\
\hline Funcionalidade & $\begin{array}{l}\text { Utilização de } \\
\text { avisos push para } \\
\text { prestação do } \\
\text { serviço }\end{array}$ & $\begin{array}{l}\text { Notificar o cidadão por } \\
\text { meio de avisos push } \\
\text { de alguma etapa da } \\
\text { prestação de serviço. } \\
\text { Exemplo 1: avisar de } \\
\text { multas de trânsito } \\
\text { recebidas. Exemplo } \\
\text { 2: enviar lembretes } \\
\text { de agendamentos } \\
\text { realizados em unidades } \\
\text { de prestação de serviço. } \\
\text { Exemplo 3: avisar os } \\
\text { valores das contas } \\
\text { de água e energia } \\
\text { recebidas. }\end{array}$ & $\begin{array}{l}\text { Centeno, } \\
\text { Andrade e } \\
\text { Souza, 2015; } \\
\text { Winkler, Ziekow } \\
\text { e Weinberg, } \\
2012\end{array}$ \\
\hline Funcionalidade & $\begin{array}{l}\text { Utilização de } \\
\text { QR Codes para } \\
\text { a prestação de } \\
\text { serviços }\end{array}$ & $\begin{array}{l}\text { Usar QR Codes (códigos } \\
\text { de resposta rápida) para } \\
\text { oferecer uma forma de } \\
\text { acesso a um conteúdo } \\
\text { específico. Esses códigos } \\
\text { podem ser usados } \\
\text { como um link em que } \\
\text { o usuário aponta o } \\
\text { dispositivo móvel, lê o } \\
\text { código e visualiza uma } \\
\text { página ou conteúdo } \\
\text { específico. }\end{array}$ & Berbe, 2014 \\
\hline
\end{tabular}




\begin{tabular}{|c|c|c|c|}
\hline Funcionalidade & $\begin{array}{l}\text { Informações real } \\
\text { time }\end{array}$ & $\begin{array}{l}\text { Apresentar informações } \\
\text { atualizadas em tempo } \\
\text { real no aplicativo } \\
\text { como as condições de } \\
\text { estradas ou o tempo } \\
\text { médio para atendimento } \\
\text { em unidades de } \\
\text { atendimento. }\end{array}$ & $\begin{array}{l}\text { Centeno, } \\
\text { Andrade e } \\
\text { Souza, } 2015\end{array}$ \\
\hline $\begin{array}{l}\text { Informações sobre } \\
\text { o aplicativo }\end{array}$ & $\begin{array}{l}\text { Atualização do } \\
\text { aplicativo }\end{array}$ & $\begin{array}{l}\text { Avaliar se o aplicativo } \\
\text { teve atualização para } \\
\text { correção de erros ou } \\
\text { inserção de melhorias no } \\
\text { ano corrente. }\end{array}$ & $\begin{array}{l}\text { Análise dos } \\
\text { aplicativos }\end{array}$ \\
\hline $\begin{array}{l}\text { Informações sobre } \\
\text { o aplicativo }\end{array}$ & $\begin{array}{l}\text { Número de } \\
\text { downloads }\end{array}$ & $\begin{array}{l}\text { Avaliar o grau de } \\
\text { disseminação do } \\
\text { aplicativo no Estado } \\
\text { utilizando como } \\
\text { referência o número de } \\
\text { usuários do serviço de } \\
\text { telefonia móvel }\end{array}$ & $\begin{array}{l}\text { Centeno, } \\
\text { Andrade e } \\
\text { Souza, 2015; } \\
\text { Análise dos } \\
\text { aplicativos }\end{array}$ \\
\hline $\begin{array}{l}\text { Informações sobre } \\
\text { o aplicativo }\end{array}$ & $\begin{array}{l}\text { Tamanho do } \\
\text { aplicativo }\end{array}$ & $\begin{array}{l}\text { Identificar o tamanho do } \\
\text { aplicativo quando está } \\
\text { instalado no celular. }\end{array}$ & $\begin{array}{l}\text { Análise dos } \\
\text { aplicativos }\end{array}$ \\
\hline $\begin{array}{l}\text { Informações sobre } \\
\text { o aplicativo }\end{array}$ & $\begin{array}{l}\text { Nota na loja do } \\
\text { aplicativo }\end{array}$ & $\begin{array}{l}\text { Identificar a nota dada } \\
\text { pelos usuários do } \\
\text { aplicativo na Play Store }\end{array}$ & $\begin{array}{l}\text { Análise dos } \\
\text { aplicativos }\end{array}$ \\
\hline $\begin{array}{l}\text { Relacionamento } \\
\text { com o cidadão }\end{array}$ & $\begin{array}{l}\text { Canal de } \\
\text { relacionamento }\end{array}$ & $\begin{array}{l}\text { Disponibilizar um canal } \\
\text { de relacionamento } \\
\text { direto no aplicativo } \\
\text { (e-mail, SMS ou link } \\
\text { para página da rede } \\
\text { social ou para avaliação } \\
\text { na loja) com o Governo } \\
\text { para enviar dúvida, } \\
\text { reclamações, sugestões } \\
\text { e elogios. }\end{array}$ & $\begin{array}{l}\text { Centeno, } \\
\text { Andrade e } \\
\text { Souza, 2015; } \\
\text { Chanana1, } \\
\text { Agrawal e Punia, } \\
2016\end{array}$ \\
\hline $\begin{array}{l}\text { Relacionamento } \\
\text { com o cidadão }\end{array}$ & $\begin{array}{l}\text { Respostas aos } \\
\text { comentários da } \\
\text { loja }\end{array}$ & $\begin{array}{l}\text { O órgão ou entidade } \\
\text { responde aos } \\
\text { comentários postados na } \\
\text { loja do aplicativo }\end{array}$ & $\begin{array}{l}\text { Análise dos } \\
\text { aplicativos }\end{array}$ \\
\hline $\begin{array}{l}\text { Segurança da } \\
\text { informação }\end{array}$ & $\begin{array}{l}\text { Políticas de } \\
\text { Privacidade }\end{array}$ & $\begin{array}{l}\text { O aplicativo apresenta as } \\
\text { políticas de privacidade } \\
\text { dos dados utilizados no } \\
\text { mesmo. }\end{array}$ & $\begin{array}{l}\text { Antovski e } \\
\text { Gusev, 2005; } \\
\text { Kushchu e } \\
\text { Kuscu, 2003; } \\
\text { Chanana1, } \\
\text { Agrawal e Punia, } \\
2016\end{array}$ \\
\hline
\end{tabular}




\begin{tabular}{|c|c|c|c|}
\hline $\begin{array}{l}\text { Segurança da } \\
\text { informação }\end{array}$ & $\begin{array}{l}\text { Identificação do } \\
\text { usuário }\end{array}$ & $\begin{array}{l}\text { Observar se há } \\
\text { instrumentos que } \\
\text { identifiquem o usuário } \\
\text { que está acessando os } \\
\text { serviços. }\end{array}$ & $\begin{array}{l}\text { Centeno, } \\
\text { Andrade e } \\
\text { Souza, 2015; } \\
\text { Alkaabi e Ayad, } \\
2016 \\
\end{array}$ \\
\hline Serviços & $\begin{array}{l}\text { Tipo de categoria } \\
\text { predominante } \\
\text { entre os serviços }\end{array}$ & $\begin{array}{l}\text { Os serviços } \\
\text { disponibilizados no } \\
\text { aplicativo podem ser } \\
\text { categorizados em: } \\
\text { serviço informativo/ } \\
\text { educativo, serviço } \\
\text { interativo, serviço } \\
\text { transacional e serviço } \\
\text { de governança e } \\
\text { participação do cidadão }\end{array}$ & $\begin{array}{l}\text { OCDE, } 2011 \text {; } \\
\text { Winkler, Ziekow } \\
\text { e Weinberg, } \\
\text { 2012; Kushchu } \\
\text { e Kuscu, 2003; } \\
\text { Chanana1, } \\
\text { Agrawal e Punia, } \\
2016\end{array}$ \\
\hline Usabilidade & $\begin{array}{l}\text { Cadastro de } \\
\text { dados }\end{array}$ & $\begin{array}{l}\text { O cidadão pode fazer } \\
\text { o cadastro prévio } \\
\text { dos seus dados para } \\
\text { utilizar posteriormente } \\
\text { no aplicativo sem a } \\
\text { necessidade de digitar } \\
\text { novamente os mesmos } \\
\text { dados. }\end{array}$ & $\begin{array}{l}\text { Análise dos } \\
\text { aplicativos }\end{array}$ \\
\hline Usabilidade & $\begin{array}{l}\text { Navegação } \\
\text { principal sempre } \\
\text { visível }\end{array}$ & $\begin{array}{l}\text { Mantenha a navegação } \\
\text { principal sempre visível. } \\
\text { O usuário deve ter } \\
\text { acesso ao menu de } \\
\text { navegação em qualquer } \\
\text { página. }\end{array}$ & Berbe, 2014 \\
\hline Usabilidade & $\begin{array}{l}\text { Facilidade } \\
\text { de acesso } \\
\text { às principais } \\
\text { funcionalidades. }\end{array}$ & $\begin{array}{l}\text { Possibilitar que os } \\
\text { usuários acessem } \\
\text { com facilidade } \\
\text { funcionalidades } \\
\text { frequentes de um } \\
\text { aplicativo por meio } \\
\text { da criação de atalhos } \\
\text { ou customização do } \\
\text { aplicativo conforme as } \\
\text { suas necessidades }\end{array}$ & $\begin{array}{l}\text { Olibário, 2013; } \\
\text { Berbe, 2014; } \\
\text { Chanana1, } \\
\text { Agrawal e Punia, } \\
2016\end{array}$ \\
\hline Usabilidade & $\begin{array}{l}\text { Padronização da } \\
\text { interface }\end{array}$ & $\begin{array}{l}\text { O aplicativo possui } \\
\text { uma padronização } \\
\text { dos elementos, cores, } \\
\text { conjunto de ícones, } \\
\text { logotipos e seções para } \\
\text { reforçar a sua identidade } \\
\text { visual. }\end{array}$ & $\begin{array}{l}\text { Olibário, 2013;; } \\
\text { Berbe, } 2014\end{array}$ \\
\hline
\end{tabular}

Fonte: Elaborado pelos autores, 2017 


\section{Resultados}

Os resultados da pesquisa são apresentados em oito categorias com a exemplificação das telas de alguns a plicativos que possuem os critérios identificados.

\section{Conteúdo}

Em relação à classificação dos serviços, sete dos dez aplicativos avaliados utilizam a classificação por assunto para organizar o conteúdo. Os aplicativos de Mato Grosso (Figura 1) e de Goiás utilizam o critério organizacional para organizar os serviços, o que pode dificultar o acesso do cidadão, já que ele pode não saber o nome do órgão responsável pela prestação do serviço. A arquitetura da informação do aplicativo não pode ser construída baseando-se na estrutura organizacional do órgão ou entidade e deve ser voltada às necessidades do usuário (BERBE, 2014).

\section{Figura 1 - Tela inicial do aplicativo MT Cidadão}

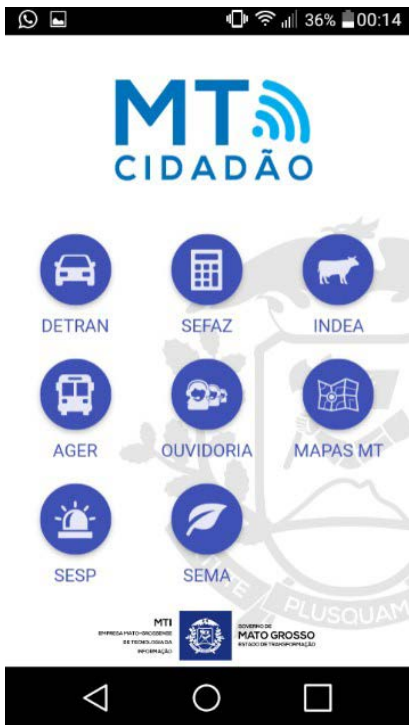

Em relação às áreas de atuação dos serviços, identificamos que existe uma variedade de vinte e cinco temas oferecidos pelos dez aplicativos avaliados. Os dois principais assuntos que foram contemplados foram "Unidades de atendimento do Governo", com sete indicações, e "Veículos e Condutores", com seis indicações. O aplicativo RS Móvel (Figura 2) foi o que apresentou a maior abrangência de serviços, contemplando quinze áreas. Temas como "Saúde" e "Educação" foram contemplados no máximo por dois aplicativos pesquisados. 
Um relatório elaborado pela União Europeia em 2017 identificou como uma boa prática na área do m-gov o Mapa do Cidadão desenvolvido pelo Governo de Portugal. Por meio desse aplicativo os cidadãos podem saber quais os serviços públicos estão mais próximos do usuário por meio de serviços geolocalizados, e quais documentos são necessários para resolver a prestação do serviço ou receber uma mensagem eletrônica informando o seu lugar na fila (UNIÃo EUROPEIA, 2017).

\section{Funcionalidades}

Poucos aplicativos utilizam as características específicas dos aplicativos móveis como recursos que utilizam geolocalização, câmeras, avisos de push e QR Codes para melhorar a comunicação com o governo. Um aplicativo que vem ganhando destaque na área pública e utiliza alguns recursos específicos citados acima é o Colab.

O Colab é uma rede social que proporciona aos cidadãos o poder de fiscalizar os serviços públicos. O cidadão pode reportar um problema identificado na cidade, como um buraco na rua. Para isso, ele pode utilizar a câmera do seu celular para tirar uma foto e utilizar o seu GPS para geolocalizar o problema. Após o cadastro da solicitação, o usuário pode compartilhá-la no Facebook para os seus amigos.

Atualmente, ele é o canal de relacionamento de 90 organizações públicas, principalmente de prefeituras. O aplicativo foi vencedor no prêmio AppMyCity! de 2013, da Organização New Cities Foundation (http://www.appmycity.org/), que anualmente elege o melhor aplicativo urbano do mundo.

Os aplicativos de Minas Gerais (Figura 2), Pará e Piauí utilizam o recurso de câmera para que cidadão anexe alguma foto do problema a ser reportado, por exemplo, em uma unidade de atendimento. 
Figura 2 - Tela para avaliação de unidades de atendimento do MG App

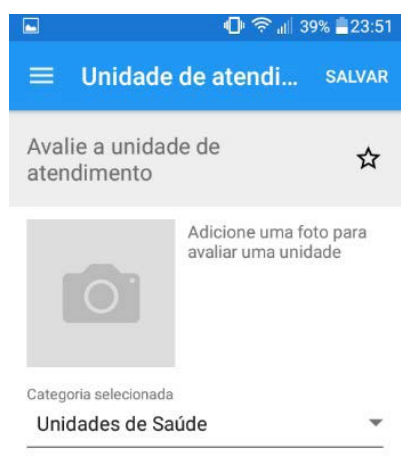

\section{Selecione uma cidade}

\section{Unidade}

\section{$\triangleleft \quad \bigcirc \quad \square$}

Em relação aos avisos de push, foram identificados que aplicativos de Minas Gerais, São Paulo e Bahia (Figura 3) enviam notificações sobre agendamentos realizados em unidades de atendimento. No caso do QR Codes, não foi identificado aplicativo que utilize esse recurso.

\section{Figura 3 - Tela de Notificação do SAC Mobile}

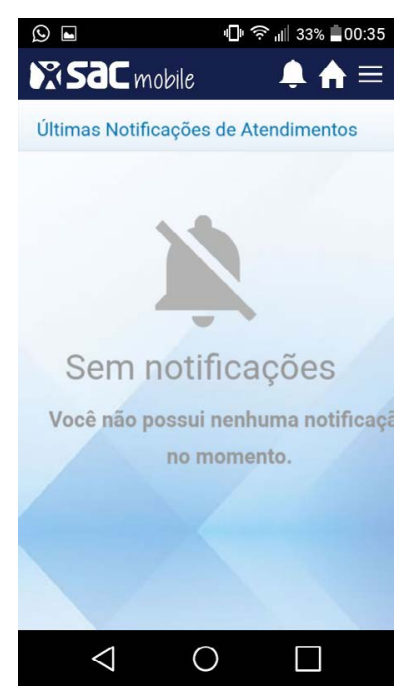

Outros recursos pouco disponibilizados pelos aplicativos foram a "busca" e "informações real time". Apenas 3 apps (Minas Gerais, Rio Grande do Sul e São Paulo) possuíam a possibilidade de o usuário realizar uma busca dos serviços e informações disponíveis. No caso das informações real time, o MG app e o SAC BA 
Mobile (Figura 4) disponibilizam o tempo de espera nas unidades de atendimento. Já o RS Móvel apresenta o movimento nas estradas gaúchas (Figura 5).

Figura 4 - Tela do tempo de espera do atendimento do SAC Mobile

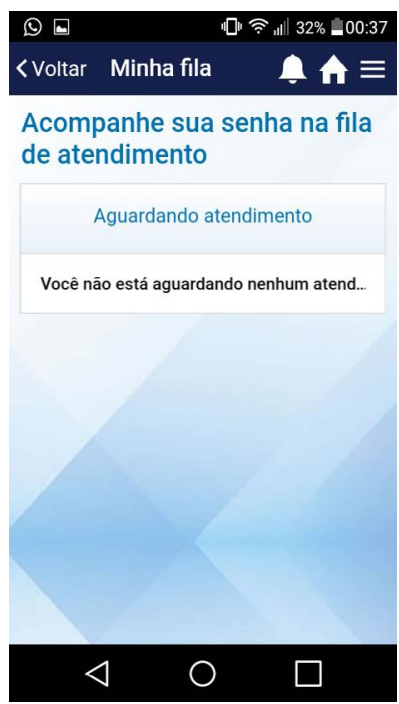

Figura 5 - Tela da situação das estradas do RS Móvel

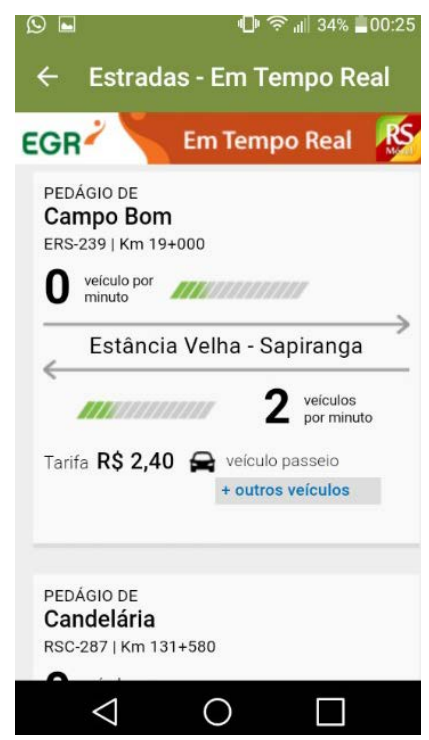

Centeno, Andrade e Souza (2015) destacam que geralmente aplicativos de governo são construídos mesmo quando não utilizam funcionalidades exclusivas de dispositivos móveis, como geolocalização, câmeras, aviso push e QR Code. Nessa análise, o aplicativo MG app é o que mais utiliza esse tipo de recurso, não 
apresentando apenas o QR Code, ainda que em pontos isolados do aplicativo, não contemplando todos os serviços. O aplicativo do Governo do Pará dispõe de geolocalização e câmera para todos os serviços disponíveis no aplicativo. Nessa situação, a experiência internacional demonstra que é possível utilizar a técnica do responsive web design, o que não necessitaria do desenvolvimento específico para aplicativos móveis (CENTENO; ANDRADE; SOUZA, 2015). O conceito de responsive web design ou design responsivo foi criado em 2010 no artigo "Responsive Web Design", escrito por Ethan Marcotte (2010) no blog "A List Apart". O autor destaca que, em vez de desenvolver um design para cada dispositivo, deveria ser projetado um único código que adaptasse o layout para as diferentes telas, por meio de tecnologias padronizadas (HyperText Markup Language [HTML] e Cascading Style Sheets [CSS]).

\section{Serviços}

Os serviços dos aplicativos estaduais são desenvolvidos predominantemente no modelo interativo, cuja principal característica é a comunicação individual com o intuito de acessar documentos personalizados e formulários. Podemos destacar com exemplos desses serviços o acesso a contas de água e energia; consulta a multas e pontuação do condutor; e consulta a dados de transparência. Na área de serviços informativos, podemos destacar a divulgação de notícias do governo e agenda de eventos institucionais.

Em relação ao tipo de modelo transacional, seis aplicativos oferecem esse tipo de serviço. Podemos exemplificar como serviços transacionais o agendamento de serviços nas unidades de atendimentos e a emissão de atestado de antecedentes criminais. No que tange aos serviços participativos, podemos destacar os que têm como objetivo auxiliar na melhoria da prestação do serviço. São exemplos desses serviços a possibilidade de avaliar as unidades de atendimento do Governo, como apresentado no MG app.

\section{Usabilidade}

No que tange aos recursos de usabilidade, a grande maioria dos aplicativos (90\%) possui padronização da interface do aplicativo de forma a manter uma identidade visual. Apenas no aplicativo do Governo de Goiás não foi identificada essa padronização. Verificou-se que sete dos dez aplicativos pesquisados possuem navegação principal sempre visível, permitindo aos cidadãos sempre ter acesso a outros serviços do aplicativo sem a necessidade de realizar vários passos.

Dois recursos que facilitam muito a navegabilidade dos cidadãos aos aplicativos para agilizar o acesso mais rápido aos serviços são pouco frequentes. O primeiro 
recurso é a possibilidade de o usuário personalizar o seu aplicativo com os serviços que ele mais utiliza. Esse recurso foi identificado apenas nos aplicativos MG app e no ES na Palma da Mão. No MG app o usuário tem a possibilidade de configurar na página inicial do aplicativo os serviços a que ele tem mais acesso (Figura 6).

Figura 6 - Página inicial do MG app

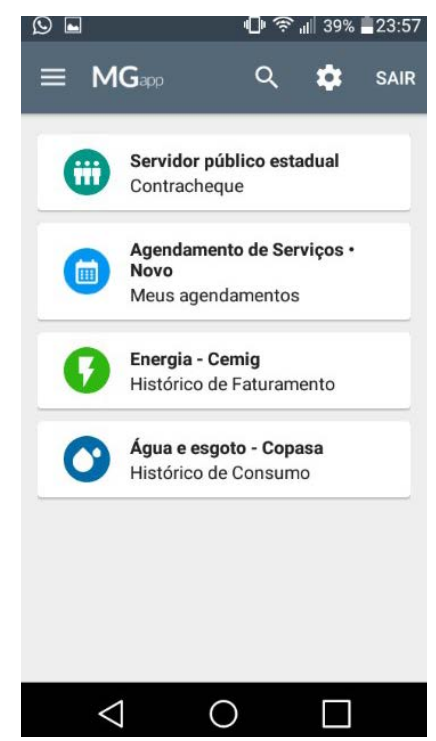

Outra opção pouco utilizada pelos aplicativos é a possibilidade de o cidadão realizar o seu cadastro e utilizar essas informações para o acesso aos serviços sem a necessidade de ter que digitar toda vez um chassi de um carro, por exemplo. Esse recurso foi identificado apenas nos aplicativos baiano, capixaba, paulista e mineiro.

Por fim, existe uma opção interessante identificada nos apps do Governo do Estado de São Paulo e do Governo do Estado de Minas Gerais que é o acesso a outros aplicativos específicos do governo. Tendo em vista que existem necessidades específicas de alguns cidadãos, não é recomendável trazer todos os serviços para um aplicativo único que poderia impactar no seu tamanho. O Governo do Estado de São Paulo possui 28 aplicativos móveis disponíveis e um aplicativo-base, denominado SP Serviços, que funciona como portal de serviços públicos, no qual está disponível o acesso para download de todas as aplicações em uso pelo Governo (Figura 7). 
Figura 7 - Tela inicial do Sp Serviços

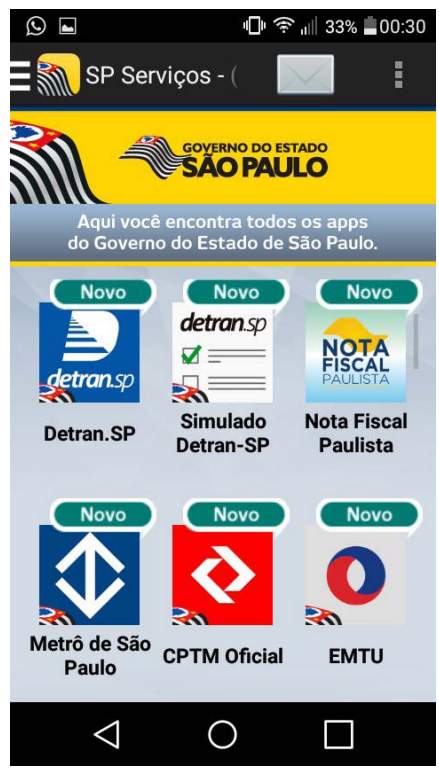

A União Europeia (2017) identificou como uma boa prática na área do m-gov o aplicativo do Governo de Malta denominado Maltapps. Esse aplicativo lançado em 2017 lista todos os aplicativos móveis do país, permitindo que os cidadãos possam localizar os serviços de m-gov oficiais do governo sem a necessidade de pesquisálos nas respectivas lojas de aplicativos. Cada serviço é classificado em doze setores representando as várias entidades e departamentos governamentais.

\section{Informações do aplicativos}

Na Play Store é possível extrair informações importantes sobre o desempenho dos aplicativos. A primeira informação que podemos destacar são as notas dadas pelos usuários em relação ao aplicativo. A nota pode variar de uma a cinco estrelas. A média dos aplicativos analisados está em 4,05 estrelas. As duas maiores são dos aplicativos dos Governos de Mato Grosso e Rio Grande do Sul, com as notas 4,5 e 4,3, respectivamente. A menor nota é do aplicativo ES na Palma da Mão com 3,5. A título de comparação, dois aplicativos de muita utilização pelos usuários, como WhatsApp e Facebook, têm notas de 4,5 e 4,0, respectivamente.

Identificou-se que $60 \%$ dos aplicativos tiveram alguma atualização neste ano para correção de erros ou implantação das melhorias. Apenas os aplicativos dos Governos de Goiás e Paraíba, que têm mais de 3 anos de existência, não apresentam qualquer atualização. Em relação ao tamanho dos aplicativos, a média é de 32,43 MB. Existem dois aplicativos com tamanhos maiores que 80 MB. São os aplicativos do Governo 
da Bahia e de Mato Grosso. Tendo em vista a limitação dos aparelhos em relação ao espaço de memória e número cada vez crescente de novos aplicativos, é interessante que os mesmos sejam desenvolvidos com tamanhos reduzidos, de forma que o usuário não tenha que desinstalar a todo o momento para instalação de novos.

Os dois aplicativos que apresentam maior número de downloads são de São Paulo e Minas Gerais. O SP Serviços apresenta entre 500.000 - 1.000.000 downloads, enquanto o MG app apresenta entre 100.000 - 500.000. Apesar de apresentarem o maior número de downloads, identifica-se ainda pouca penetração na população como um todo. No caso de São Paulo, representa entre 1 e $2 \%$ da população, enquanto em Minas Gerais representa entre 0,5 e 2,5\% (BRASIL, 2017).

\section{Relacionamento com o cidadão}

No que tange ao relacionamento com o cidadão, seis aplicativos possuem um canal direto para envio de dúvidas, reclamações, sugestões e elogios para o governo pelo próprio aplicativo. Entretanto, apenas os aplicativos dos Governos da Bahia, Espírito Santo, Minas Gerais e São Paulo respondem aos comentários postados na loja do Play Store pelos usuários referentes a dúvidas, reclamações ou elogios sobre o aplicativo. A existências desses canais e a resposta pelos governos aprimora a relação com o cidadão.

\section{Segurança}

A maioria dos aplicativos estaduais (seis aplicativos) utiliza um meio para identificação do cidadão. Essa possibilidade, apesar de inserir uma etapa adicional para o cidadão acessar o serviço, auxilia os governos a entenderem de forma personalizada as necessidades de cada cidadão. Apenas quatro aplicativos (ES na Palma da Mão, MG app, Governo Digital Pará e SP Serviços) apresentam políticas de privacidade dos dados fornecidos pelos cidadãos. Essa política é necessária para manter uma relação transparente entre governo e cidadão.

\section{Ajuda e documentação}

A grande maioria dos aplicativos estaduais (70\%) não possui uma seção de ajuda nem apresenta o termo de uso de utilização do app. Os aplicativos dos Governos de Minas Gerais e Espírito Santo são os únicos que possuem ajuda e termo de uso. O app do governo paulista possui ajuda e o do gaúcho possui termo de uso. A falta da seção ajuda no aplicativo dificulta o esclarecimento de dúvidas frequentes sobre 
a utilização do mesmo. Da mesma forma, é importante que o usuário esteja ciente das regras de utilização do aplicativo por meio do termo de uso.

\section{Considerações finais}

Os resultados da avaliação demonstram que os estados e o Distrito Federal ainda possuem um longo caminho a percorrer para aproveitar as potencialidades do $m$-gov. Tendo em vista a expansão do número de dispositivos móveis com acesso a dados, isso demonstra que esse tema deverá ser discutido cada vez mais pelos governos nacionais e internacionais.

Apenas 10 estados dos 27 pesquisados, incluindo o Distrito Federal, possuem um aplicativo que centraliza os serviços dos governos. O número de downloads dos aplicativos também representa uma parcela pequena da população, tendo em vista o número de usuários potenciais.

Poucos serviços utilizam-se dos recursos tecnológicos dos dispositivos móveis, como GPS, câmera e avisos por push. Dessa forma, cabe uma reflexão se é necessário o desenvolvimento de um aplicativo específico ou adaptação dos portais para um design responsivo para essas tecnologias.

Os gestores estaduais devem se preocupar com a elaboração de uma estratégia para contemplar o $m$-gov no atendimento ao cidadão considerando as melhores práticas internacionais no tema. Organizações internacionais com a União Europeia e as Organizações das Nações Unidades têm publicado estudos recentes e recorrentes sobre essas melhores práticas.

\section{Referências bibliográficas}

ALKAABI, Saif Obaid; AYAD, Nabil. Factors affecting m-government deployment and adoption. World Academy of Science, Engineering and Technology, International Journal of Social, Behavioral, Educational, Economic, Business and Industrial Engineering, v. 10, n. 1, p. 314-322, 2016.

AlLAZO, Edwar Andres Velarde; SABLón, Vicente Idalberto Becerra Sablón; IANO, Yuzo. Aplicações Governamentais para TV Digital Móvel usando Ginga NCL. In: Revista de Radiodifusão - v. 03, n. 03, set. de 2009. Disponível em: <http://www. set.com.br/revistaeletronica/radiodifusao/index.php/revistaderadiodifusaoset/ article/view/17/18 >. Acesso em: 09 de abril de 2016.

ANTOVSKI, L. GUSEV, M. M-Government Framework. In: Euro mGov. p. 36-44, 2005. ARAúso, Wagner Frederico Gomes de. A avaliação de sítios governamentais como um instrumento para melhoria dos serviços e informações on-line: da concepção aos resultados. Belo Horizonte, 2005. Disponível em: <http://portal-homologa. prodemge.gov.br/governanca/download?arquivo=194>. Acesso em: 09 de abril de 2016. 
Berbe, Alexandre Campos. Proposta de diretrizes de usabilidade para sites e aplicativos de governo eletrônico móvel. month, p. 81, 2014.

BRASIL. Instituto Brasileiro de Geografia e Estatística. Pesquisa nacional por amostra de domicílios: síntese de indicadores 2015. Rio de Janeiro: IBGE, 2016.

BRASIL. Instituto Brasileiro de Geografia e Estatística. Estimativas da população residente para os municípios e para as unidades da federação brasileiros com a data de referência em 1ㅇ de julho de 2017. Rio de Janeiro: IBGE, 2017.

CARVALHo, Lucas. Android cresce no Brasil e aumenta distância para iOS e Windows Phone, 03/05/2017. Disponível em: <https://olhardigital.uol.com.br/noticia/ android-cresce-no-brasil-e-aumenta-distancia-para-ios-e-windows-phone/68023>. Acesso em: 25 de maio de 2017.

CASTELls, Manuel. A era da informação: economia, sociedade e cultura. A sociedade em rede. São Paulo: Paz e Terra, v. 1, 7o edição, 2003.

Centeno, F. P. R.; Andrade, M. A.; SouzA, R. O. Melhoria da comunicação e do relacionamento entre o governo e os cidadãos com aplicativos móveis: estudo de caso do m-government do Estado de São Paulo. 2015. Dissertação (Mestrado Profissional em Gestão e Políticas Públicas)-Escola de Administração de Empresas de São Paulo da Fundação Getúlio Vargas, São Paulo, 2015(em fase de elaboração). Chanana, Lovneesh; Agrawal, Rajat; PuniA, Devendra Kumar. Service quality parameters for mobile government services in India. Global Business Review, v. 17, n. 1, p. 136-146, 2016.

CHEN, Zhen-Jiao; Vogel, Douglas; WANG, Zhao-Hua. How to satisfy citizens? Using mobile government to reengineer fair government processes. Decision Support Systems, v. 82, p. 47-57, 2016.

FERGUSON, Martin. Estratégias de governo eletrônico: o cenário internacional em desenvolvimento. In: EISENBERG, José; CEPIK, Marco (org). Internet e política. Belo Horizonte: Editora UFMG, 2002.

KUSHCHU, Ibrahim; KUSCU, M. Halid. From E-government to M-government: Facing the Inevitable. In: European Conference on e-Government (ECEG), 3ㅇd,, Dublin, Ireland. Trinity College, 2003.

MARCotTE, E. Responsive Web Design, 25/05/2010. Disponível em:<http://alistapart. com/article/responsive-web-design>. Acesso em: 13 de junho de 2018.

Medeiros, Paulo Henrique Ramos; GuimarãES, Tomás de Aquino. O estágio do governo eletrônico no Brasil em relação ao contexto mundial. In: Revista do Serviço Público, ano 55, Brasília, 2004. Disponível em: < http://repositorio.enap. gov.br/bitstream/handle/1/1475/2004\%20Vol.55\%2cn.1e2\%20Medeiros\%20e\%20 Guimar\%C3\%A3es.pdf?sequence=1\&isAllowed=y>. Acesso em: 10 de abril de 2016. MEDEIROS, Paulo Henrique Ramos; GuIMARÃES, Tomás deAquino.Ainstitucionalização do governo eletrônico no Brasil. In: Revista de Administração de Empresas (RAE), v. 46, n. 4, out-dez 2006. Disponível em: <http://rae.fgv.br/rae/vol46-num4-2006/ institucionalizacao-governo-eletronico-no-brasil>. Acesso em: 10 de abril de 2016. 
OCDE, Organização para a Cooperação e Desenvolvimento Econômico (International Telecommunication Union). M-Government: Mobile Technologies for Responsive Governments and Connected Societies, OECD Publishing. Disponível em: http:// dx.doi.org/10.1787/9789264118706-en, 2011.

OlıBárıo, José Machado Neto. Usabilidade da interface de dispositivos móveis: heurísticas e diretrizes para o design / Olibário José Machado Neto; orientadora Maria da Graça Campos Pimentel. -- São Carlos, 2013. 118 p. Dissertação (Mestrado - Programa de Pós-Graduação em Ciências de Computação e Matemática Computacional) -Instituto de Ciências Matemáticas e de Computação, Universidade de São Paulo, 2013.

ORgANIZAÇÃO DAS NAÇÕES UNIDAS. Department of Economic and Social Affairs. United Nations E-Government Survey 2016: E-Government in Support of Sustainable Development 2016. Nova lorque, 2016.

PIMENTEL, Danton Sousa. Desenvolvimento e Avaliação de uma Aplicação de m-Gov. Monografia apresentada ao Curso de Graduação em Ciência da Computação, Departamento de Ciência da Computação, Instituto de Matemática, Universidade Federal da Bahia. Salvador, 2009.

WINKLER, T. J.; ZIEKOW, H.; WeINBERG, M. Municipal Benefits of Participatory Urban Sensing: A Simulation Approach and Case Validation. In: Journal of Theoretical and Applied Electronic Commerce Research, v. 7, n.3, p. 101-120 - dez 2012.

UNIÃo EUROPEIA. Comissão Europeia. eGovernment Benchmark 2017: Taking stock of user-centric design and delivery of digital public services in Europe. 2017.

\section{Rodrigo Diniz Lara}

Possui mestrado em Administração pela Universidade Federal de Minas Gerais (UFMG). Atualmente é Superintendente Central de Governança Eletrônica da Secretaria de Estado de Planejamento e Gestão de Minas Gerais. Contato: rdinizl@hotmail.com

\section{Marlusa Gosling}

Possui doutorado em Administração pela Universidade Federal de Minas Gerais (UFMG) e posdoc em Gestão do Turismo na Universidade de Algarve, Portugal. Atualmente é professora de Marketing da UFMG, Coordenadora do Núcleo de Estudos e Estratégias em Comunicação Integrada de Marketing e Turismo (NEECIM-TUR) e Coordenadora da Pós-Graduação Lato Sensu do Centro de Pós-Graduação e Pesquisas em Administração da UFMG, curso de Gestão Estratégica. Contato: mg.ufmg@gmail.com

Izabela França Rodrigues

Possui mestrado em Administração Pública pela Fundação João Pinheiro (FJP). Atualmente é Especialista em Políticas Públicas e Gestão Governamental na Secretaria de Estado de Planejamento e Gestão de Minas Gerais. Contato: izabelarodrigues@yahoo.com.br 
APÊNDICE A - Tabela de Análise dos Aplicativos Estaduais e do Distrito Federal

\begin{tabular}{|c|c|c|c|c|c|c|c|c|}
\hline \multirow[b]{2}{*}{$\begin{array}{l}\text { Critério/ } \\
\text { Estado }\end{array}$} & \multicolumn{2}{|c|}{$\begin{array}{c}\text { Ajuda e } \\
\text { documentação }\end{array}$} & \multicolumn{6}{|c|}{ Funcionalidades } \\
\hline & Ajuda & $\begin{array}{l}\text { Termos } \\
\text { de uso }\end{array}$ & Busca & $\begin{array}{c}\text { Câmeras } \\
\text { para } \\
\text { presta- } \\
\text { ção do } \\
\text { serviço }\end{array}$ & $\begin{array}{l}\text { GPS para } \\
\text { presta- } \\
\text { ção do } \\
\text { serviço }\end{array}$ & $\begin{array}{c}\text { Avisos } \\
\text { push para } \\
\text { prestação } \\
\text { do serviço }\end{array}$ & $\begin{array}{c}\text { Utiliza- } \\
\text { ção } \\
\text { de QR } \\
\text { Codes }\end{array}$ & $\begin{array}{l}\text { Informa- } \\
\text { ções real } \\
\text { time }\end{array}$ \\
\hline Bahia (SAC BA & & & & & & & & \\
\hline Mobile) & Não & Não & Não & Não & Sim & Sim & Não & Sim \\
\hline $\begin{array}{l}\text { Espiríto Santo } \\
\text { (ES na Palma } \\
\text { da Mão) }\end{array}$ & Sim & Sim & Não & Não & Não & Não & Não & Não \\
\hline $\begin{array}{l}\text { Goiás } \\
\text { (VaptVupt) }\end{array}$ & Não & Não & Não & Não & Não & Não & Não & Não \\
\hline $\begin{array}{l}\text { Mato Grosso } \\
\text { (MT Cidadão) }\end{array}$ & Não & Não & Não & Não & Sim & Não & Não & Não \\
\hline $\begin{array}{l}\text { Minas Gerais } \\
\text { (MG app) }\end{array}$ & Sim & Sim & Sim & Sim & Sim & Sim & Não & Sim \\
\hline $\begin{array}{l}\text { Pará } \\
\text { (Governo } \\
\text { Digital Pará) }\end{array}$ & Não & Não & Não & Sim & Sim & Não & Não & Não \\
\hline $\begin{array}{l}\text { Paraíba } \\
\text { (Governo da } \\
\text { Paraíba) }\end{array}$ & Não & Não & Não & Não & Sim & Não & Não & Não \\
\hline $\begin{array}{l}\text { Piauí (Gestão } \\
\text { Transparente) }\end{array}$ & Não & Não & Não & Sim & Sim & Não & Não & Não \\
\hline $\begin{array}{l}\text { Rio Grande } \\
\text { do Sul (RS } \\
\text { Móvel) }\end{array}$ & Não & Sim & Sim & Não & Não & Não & Não & Sim \\
\hline $\begin{array}{l}\text { São Paulo (SP } \\
\text { Serviços) }\end{array}$ & Sim & Não & Sim & Não & Não & Sim & Não & Não \\
\hline
\end{tabular}




\begin{tabular}{|c|c|c|c|c|c|c|c|c|}
\hline \multirow[b]{2}{*}{$\begin{array}{l}\text { Critério/ } \\
\text { Estado }\end{array}$} & \multicolumn{4}{|c|}{ Informações do aplicativo } & \multicolumn{2}{|c|}{$\begin{array}{c}\text { Relacionamento } \\
\text { com o Cidadão }\end{array}$} & \multicolumn{2}{|c|}{$\begin{array}{l}\text { Segurança da } \\
\text { Informação }\end{array}$} \\
\hline & $\begin{array}{l}\text { Atualização } \\
\text { do aplicativo }\end{array}$ & $\begin{array}{c}\text { Número } \\
\text { de down- } \\
\text { loads }\end{array}$ & $\begin{array}{l}\text { Tama- } \\
\text { nho do } \\
\text { aplica- } \\
\text { tivo }\end{array}$ & $\begin{array}{c}\text { Nota } \\
\text { na } \\
\text { loja } \\
\text { do } \\
\text { aplica- } \\
\text { tivo }\end{array}$ & $\begin{array}{l}\text { Canal } \\
\text { de rela- } \\
\text { ciona- } \\
\text { mento }\end{array}$ & $\begin{array}{c}\text { Respostas } \\
\text { aos co- } \\
\text { mentários } \\
\text { da loja }\end{array}$ & $\begin{array}{c}\text { Políticas } \\
\text { de } \\
\text { Priva- } \\
\text { cidade }\end{array}$ & $\begin{array}{c}\text { Identifi- } \\
\text { cação } \\
\text { do } \\
\text { usuário }\end{array}$ \\
\hline $\begin{array}{l}\text { Bahia (SAC } \\
\text { BA Mobile) }\end{array}$ & $27 / 01 / 2017$ & $\begin{array}{c}50.000 \\
-100.000 \\
\end{array}$ & 85,41 & 4,1 & $\operatorname{Sim}$ & $\operatorname{Sim}$ & Não & Sim \\
\hline $\begin{array}{l}\text { Espiríto } \\
\text { Santo (ES } \\
\text { na Palma } \\
\text { da Mão) }\end{array}$ & $21 / 03 / 2017$ & $\begin{array}{c}5.000 \\
-10.000\end{array}$ & 19,57 & 3,5 & Sim & Sim & Sim & Sim \\
\hline $\begin{array}{l}\text { Goiás } \\
\text { (VaptVupt) }\end{array}$ & $25 / 02 / 2014$ & $\begin{array}{c}10.000 \\
-50.000 \\
\end{array}$ & 3,3 & 4,1 & Não & Não & Não & Não \\
\hline $\begin{array}{l}\text { Mato } \\
\text { Grosso } \\
\text { (MT } \\
\text { Cidadão) }\end{array}$ & $28 / 03 / 2017$ & $\begin{array}{c}10.000 \\
-50.000\end{array}$ & 88,16 & 4,5 & Não & Não & Não & Não \\
\hline $\begin{array}{l}\text { Minas } \\
\text { Gerais } \\
\text { (MG app) }\end{array}$ & $25 / 04 / 2017$ & $\begin{array}{c}100.000 \\
-500.000\end{array}$ & 47,68 & 4,1 & Sim & Sim & Sim & Sim \\
\hline $\begin{array}{l}\text { Pará } \\
\text { (Governo } \\
\text { Digital } \\
\text { Pará) } \\
\end{array}$ & $02 / 03 / 2017$ & $\begin{array}{c}5.000 \\
-10.000\end{array}$ & 19,76 & 3,7 & Não & Não & Sim & Sim \\
\hline $\begin{array}{l}\text { Paraíba } \\
\text { (Governo } \\
\text { da Paraíba) }\end{array}$ & 04/04/2014 & $\begin{array}{r}5.000 \\
-10.000 \\
\end{array}$ & 4,98 & 4,2 & Não & Não & Não & Não \\
\hline $\begin{array}{l}\text { Piauí } \\
\text { (Gestão } \\
\text { Transpa- } \\
\text { rente) }\end{array}$ & $02 / 08 / 2016$ & $\begin{array}{c}500 \\
-1000\end{array}$ & 20,1 & 3,9 & Sim & Não & Não & Sim \\
\hline $\begin{array}{l}\text { Rio Grande } \\
\text { do Sul (RS } \\
\text { Móvel) }\end{array}$ & $27 / 10 / 2015$ & $\begin{array}{c}10.000 \\
-50.000\end{array}$ & 6,19 & 4,3 & Sim & Não & Não & Não \\
\hline $\begin{array}{l}\text { São } \\
\text { Paulo (SP } \\
\text { Serviços) }\end{array}$ & $17 / 04 / 2017$ & $\begin{array}{l}500.000- \\
1.000 .000\end{array}$ & 29,48 & 4,1 & $\operatorname{Sim}$ & $\operatorname{Sim}$ & $\operatorname{Sim}$ & Sim \\
\hline
\end{tabular}




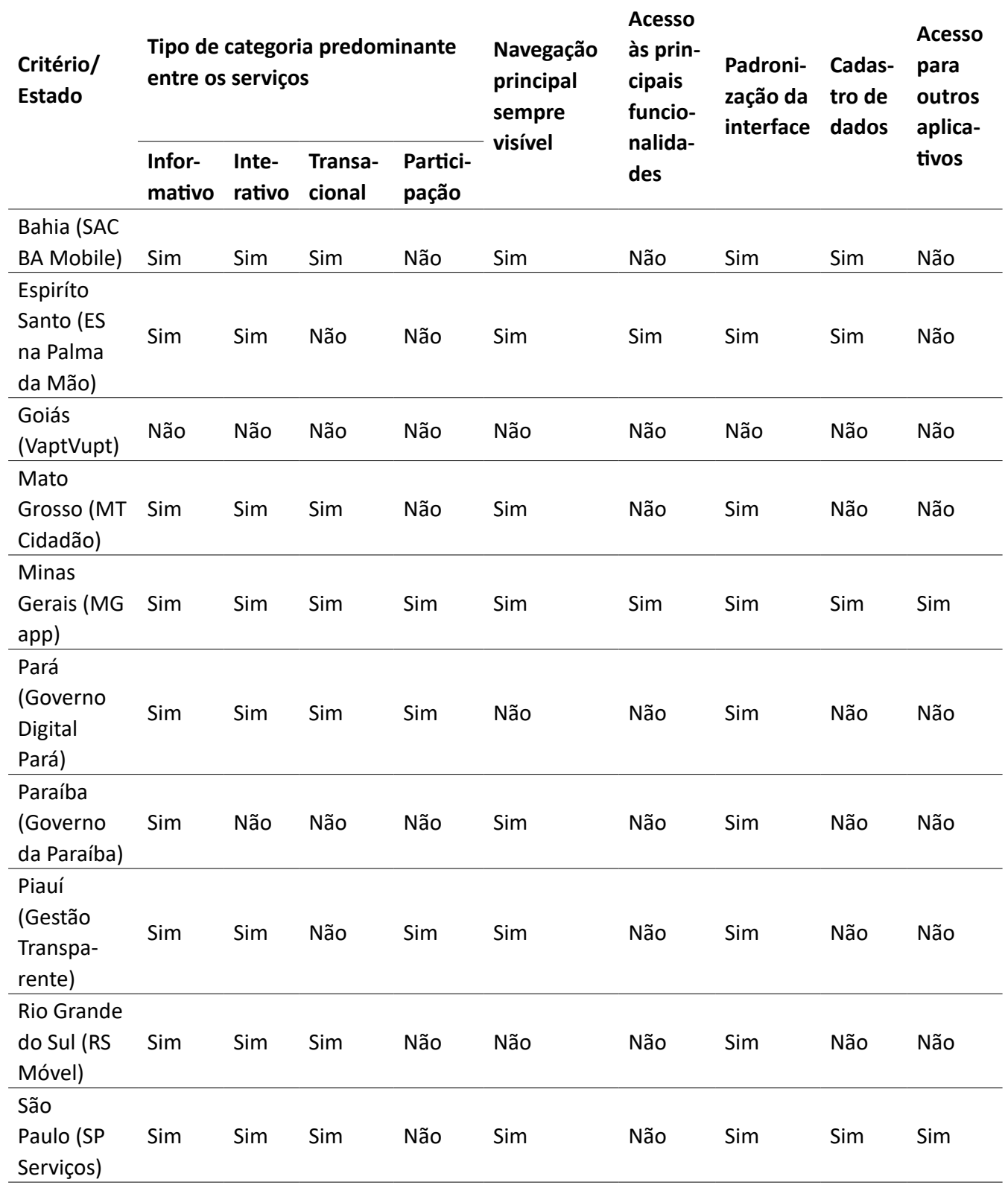




\begin{tabular}{|c|c|c|c|c|c|c|c|c|c|c|}
\hline \multirow{3}{*}{$\begin{array}{l}\text { Critério/ } \\
\text { Estado }\end{array}$} & \multicolumn{10}{|c|}{ Conteúdo } \\
\hline & \multicolumn{10}{|c|}{ Áreas de atuação } \\
\hline & $\begin{array}{c}\text { Identifi- } \\
\text { cação } \\
\text { Civil }\end{array}$ & $\begin{array}{l}\text { Acha- } \\
\text { dos e } \\
\text { Perdi- } \\
\text { dos }\end{array}$ & Trabalho & $\begin{array}{l}\text { Segu- } \\
\text { rança }\end{array}$ & $\begin{array}{l}\text { Água e } \\
\text { esgoto }\end{array}$ & Saúde & Energia & $\begin{array}{l}\text { Veícu- } \\
\text { los e } \\
\text { condu- } \\
\text { tores }\end{array}$ & $\begin{array}{l}\text { Ouvi- } \\
\text { doria }\end{array}$ & $\begin{array}{l}\text { Uni- } \\
\text { dades }\end{array}$ \\
\hline $\begin{array}{l}\text { Bahia } \\
\text { (SAC BA } \\
\text { Mobile) }\end{array}$ & $x$ & & $x$ & & & & & $x$ & $x$ & $x$ \\
\hline $\begin{array}{l}\text { Espiríto } \\
\text { Santo (ES } \\
\text { na Palma } \\
\text { da Mão) }\end{array}$ & & & & & & & & $x$ & & \\
\hline $\begin{array}{l}\text { Goiás } \\
\text { (VaptVupt) }\end{array}$ & & & & & & & & & & \\
\hline $\begin{array}{l}\text { Mato } \\
\text { Grosso } \\
\text { (MT } \\
\text { Cidadão) }\end{array}$ & $x$ & & & $\mathrm{x}$ & & & & $x$ & $x$ & $x$ \\
\hline $\begin{array}{l}\text { Minas } \\
\text { Gerais } \\
\text { (MG app) }\end{array}$ & $x$ & & $x$ & $x$ & $x$ & $x$ & $x$ & $x$ & & $x$ \\
\hline $\begin{array}{l}\text { Pará } \\
\text { (Governo } \\
\text { Digital } \\
\text { Pará) }\end{array}$ & & & & $x$ & $\mathrm{x}$ & & & & & $x$ \\
\hline $\begin{array}{l}\text { Paraíba } \\
\text { (Governo } \\
\text { da } \\
\text { Paraíba) }\end{array}$ & & & & & & & & & & $x$ \\
\hline $\begin{array}{l}\text { Piauí } \\
\text { (Gestão } \\
\text { Transpa- } \\
\text { rente) }\end{array}$ & & & & & & & & & & \\
\hline $\begin{array}{l}\text { Rio } \\
\text { Grande } \\
\text { do Sul (RS } \\
\text { Móvel) }\end{array}$ & & & & $x$ & $x$ & $x$ & $x$ & $x$ & $x$ & $x$ \\
\hline $\begin{array}{l}\text { São } \\
\text { Paulo (SP } \\
\text { Serviços) }\end{array}$ & $x$ & $x$ & $x$ & $x$ & & & & $x$ & & $x$ \\
\hline
\end{tabular}




\section{Conteúdo}

Critério/

Áreas de atuação

Estado

\begin{tabular}{|c|c|c|c|c|c|c|c|c|}
\hline Servidor & $\begin{array}{c}\text { Pesquisa } \\
\text { de } \\
\text { Processos }\end{array}$ & $\begin{array}{l}\text { Edu- } \\
\text { cação }\end{array}$ & $\begin{array}{l}\text { Legis- } \\
\text { lação }\end{array}$ & Empresa & $\begin{array}{l}\text { Diário } \\
\text { Oficial }\end{array}$ & Tributos & $\begin{array}{l}\text { Agricul- } \\
\text { tura }\end{array}$ & Animais \\
\hline
\end{tabular}

Bahia

(SAC BA

Mobile)

Espiríto

Santo (ES

na Palma

$\mathrm{X}$

$\mathrm{X}$

da Mão)

Goiás

(VaptVupt)

Mato

Grosso

(MT

Cidadão)

Minas

Gerais

X

(MG app)

Pará

(Governo

Digital

Pará)

Paraíba

(Governo

da

Paraíba)

Piauí

(Gestão

Transpa-

rente)

Rio

Grande

do Sul (RS

$\mathrm{X}$

$\mathrm{X}$

$\mathrm{X}$

$\mathrm{X}$

X

X

Móvel)

São

Paulo (SP

Serviços) 


\section{Conteúdo}

Critério/

Áreas de atuação

Conteúdo

Estado

classifica-

dos por

assunto

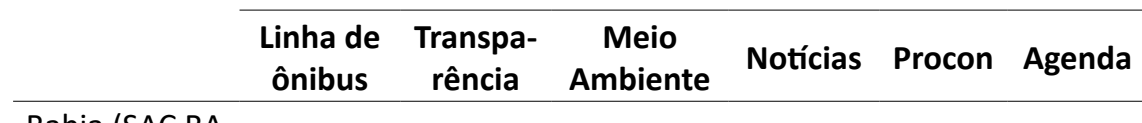

\begin{tabular}{|c|c|c|c|c|c|c|c|}
\hline $\begin{array}{l}\text { Bahia (SAC BA } \\
\text { Mobile) }\end{array}$ & & & & $x$ & & & Sim \\
\hline $\begin{array}{l}\text { Espiríto Santo } \\
\text { (ES na Palma } \\
\text { da Mão) }\end{array}$ & $x$ & $x$ & & $x$ & & $x$ & Sim \\
\hline $\begin{array}{l}\text { Goiás } \\
\text { (VaptVupt) }\end{array}$ & & & & & & & Não \\
\hline $\begin{array}{l}\text { Mato Grosso } \\
\text { (MT Cidadão) }\end{array}$ & $x$ & & $x$ & & & $x$ & Não \\
\hline $\begin{array}{l}\text { Minas Gerais } \\
\text { (MG app) }\end{array}$ & & & & & & & Sim \\
\hline $\begin{array}{l}\text { Pará (Governo } \\
\text { Digital Pará) }\end{array}$ & & & $x$ & & $x$ & & Não \\
\hline $\begin{array}{l}\text { Paraíba } \\
\text { (Governo da } \\
\text { Paraíba) }\end{array}$ & & & & $x$ & & & Sim \\
\hline $\begin{array}{l}\text { Piauí (Gestão } \\
\text { Transparente) }\end{array}$ & & $x$ & & $x$ & & & Sim \\
\hline $\begin{array}{l}\text { Rio Grande } \\
\text { do Sul (RS } \\
\text { Móvel) }\end{array}$ & & $x$ & & & & $x$ & Sim \\
\hline $\begin{array}{l}\text { São Paulo (SP } \\
\text { Serviços) }\end{array}$ & & & & & & & Sim \\
\hline
\end{tabular}

